\title{
Influences on Pressure Releasing by Blasting Breaking Hard Roof
}

\author{
Wei Zhang, Yunliang Tan, Weiyao Guo, Shitan Gu, Dianrui Mu, Shanchao Hu \\ Shandong University of Science and Technology \\ Qingdao 266590, China \\ e-mail:929031513@qq.com
}

\begin{abstract}
In order to optimize and upgrade the pressure releasing effect of blasting breaking hard roof, influence factors of it was analyzed by theoretical analysis, numerical simulation and field practice. Firstly, the impact on charge structure (charge uncoupling coefficient $K$, charge axial coefficient $l_{e}$ and charge radius $r_{b}$ ) was analyzed by indexes of fracture zone radius and crush zone radius. Secondly, the evolution trend of pressure releasing effect of different blasting parameters (angle of blasting hole, length of blasting hole and explosive charge) was studied by numerical simulation. Results show that blasting effect improves obviously with increase of charge radius and explosive charge. Reducing charge uncoupling coefficient $K$ under the premise of using uncoupling charge ways and taking axial continuous charge way are beneficial for improving blasting effect. When the angle of blasting hole is $45^{\circ}$ and it penetrates hard roof rock, pressure releasing effect is better. Combined with the construction equipment and materials in a mine, the charge structure and blasting parameters were designed, and a good application effect was got
\end{abstract}

Keywords - Hard Roof; Blasting Breaking Roof; Pressure Releasing Effect; Charge Structure; Blasting Parameters

\section{INTRODUCTION}

Rock burst can easily cause large losses as one of the major disasters in coal mine [1]. Hard roof is one of the important factors because large area will hang when the roof has strong lithology and good integrity after coal seam mining and it is not easily to cave naturally in short term. As the longwall face advances, the roof will fracture in front of coal wall, which can easily lead to rock burst [2-4]. Improving the roof condition is one of the important means to reduce the times and the intensity of rock burst [5].

A large number of engineering applications show that deep hole blasting breaking roof is one of the effective measures to improve roof conditions and prevent the rock burst caused by hard roof [6-8].Scholars analyzed stress distribution rule of surrounding rock before and after pressure releasing through numerical simulation, which provides good references for the study of blasting pressure releasing of rock burst prevention $[9,10]$. The mechanism of deep hole blasting breaking roof was discussed and its practice for rock burst prevention and control was carried by Qi[11]. Zhao [12] and Wei [13] analyzed the blasting effect under different blasting parameters (the angle of blasting hole and charge length) and different mining conditions. The charge structure of blasting hole influenced the effect of blasting pressure releasing obviously, but the research on the charge structure influencing blasting effect was not perfect, and the design process of the blasting parameters has a few weaknesses.

Based on the 3208 working face, the influence of charge structures and blasting parameters on the pressure releasing effect was analyzed through theoretical analysis and numerical simulation. Combined with the construction conditions in mine, the blasting parameters and construction technology were designed. The effect of rock burst prevention was obvious.

\section{ENGINEERING BACKGROUND}

The main coal seam, which was $3.9 \mathrm{~m}$ in thickness and has simple structures, was $3 \#$ coal seam in 3208 working face. The $1.5 \mathrm{~m}$ height of coal seam below roof was soft and broken. The Platts hardness of coal seam was $f=2 \sim 3$. The mining depth was $630 \mathrm{~m}$ and the slope length was $120 \mathrm{~m}$. Generally, the 3208 working face was a monoclinal structure. The dip angle of the coal seam was $2 \sim 10^{\circ}$, and the average value was $6^{\circ}$. The immediate roof was $2.05 \mathrm{~m}$ mudstone, and the basic roof was $21.83 \mathrm{~m}$ fine-grained sandstone, which consists of feldspar, quartz and mica and the Platts hardness was $f=7 \sim 8$. The immediate and basic floors were siltstone and sandstone, whose thickness were $1.37 \mathrm{~m}$ and $2.60 \mathrm{~m}$, respectively.

During the preliminary mining process, one rock burst occurred due to the caving of basic roof, thus blasting to break basic roof for pressure relief in 3208 roadway is essential

\section{THE TECHNOLOGY PRINCIPLE OF BLASTING BREAKING ROOF TO RELIEF PRESSURE}

The shock wave, detonation gas and strong disturbance caused by blasting can lead to coal and rock damage. Therefore, mechanical properties of coal and rock are weakened. Its ability of accumulating high stress and storing high elastic deformation energy, and the stress gradient of the axial direction are reduced. The stress condition and energy transfer condition of rock burst occurrence is mitigated [11], in other words, the occurrence probability of rock burst was reduced. The principle of blasting breaking roof is making full use of shock compression effects of the shock wave and the crack effect of stress wave, leading to the roof fractured and continuity damaged. According to the damage characteristics 
of rock after blasting, it can be divided into crush zone, fracture zone and vibration zone, as shown in fig. 1 .

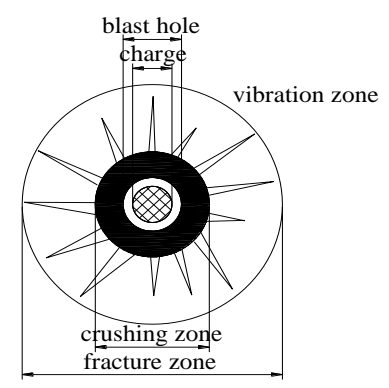

Fig.1. blasting damage effect

The rock fracture starts at the blasting of charge containing in the hole, and the gas from burst impacts on the hole wall instantaneously. Pressure of blast pulse close to the hole greatly exceeds the strength of the rock, which looks like the nature of fluid. The area is crushed by the energy of pressure pulses, which is called the crush zone. The radius of crush zone is $R_{\mathrm{c}}$, which can be expressed as formula (1) [8].

$$
R_{c}=\left[\frac{\rho_{0} D_{v}^{2} n K^{-2 \eta} l_{e} B}{8 \sqrt{2 \sigma_{c d}}}\right]^{\frac{1}{\alpha}} r_{b}
$$

When the crush zone formed, the shock wave attenuates to stress wave and spreads around the hole. The fissure zone is formed in coal connected to crush zone, which can easily lead to fracture damage called fracture zone. Fracture zone is the major functional area during the blasting process. The radius of fracture zone $R_{p}$ can be represented by formula (2).

$$
R_{p}=\left[\frac{\sigma_{c d}}{\sigma_{t d}}\right]^{\frac{1}{\beta}}\left[\frac{\rho_{0} D_{v} n K^{-2 \eta} l_{e} B}{8 \sqrt{2 \sigma_{c d}}}\right]^{\frac{1}{\alpha}} r_{b}
$$

In the area outside of the fracture zone, the stress wave turns into sound wave or strain wave, which is less than the compressive strength of the rock. It comes into the rock without damage along the radial, until attenuates to less than the tensile strength of the rock. In addition, if the free surface is close to the blasting center, it will cause stress concentration of the hoop leading to radial fracture, and extending to the free surface. This area is called vibration zone, and the radius of vibration area $R_{s}$ can be expressed as follows (3) [12] :

$$
R_{s}=(1.5 \sim 2.0) \sqrt[3]{q}
$$

In type (1), (2), (3) : $\alpha, \beta$ is shock wave attenuation index and stress wave attenuation index respectively, $\alpha=\frac{2+\mu_{d}}{1-\mu_{d}}, \quad \beta=\frac{2-\mu_{d}}{1-\mu_{d}}, \rho_{0}$ is the density of explosive, $\mathrm{kg} / \mathrm{m}^{3} ;$ $D_{v}$ is detonation velocity of explosive in coal seam, $\mathrm{m} / \mathrm{s} ; K$ is radial charge uncoupling coefficient, $K=d_{b} / d_{c}$; The $d_{b}$ and $d_{c}$ are blast hole radius and charge radius; $l_{e}$ is the charge axial coefficient, $l_{e}=l_{c} /\left(l_{b}-l_{s}\right)$. Where, $l_{\mathrm{c}}$ is the length of charge in blast hole. $l_{\mathrm{b}}$ is the length of blast hole. $l_{\mathrm{s}}$ is the sealing length in hole, $l_{e}=1$ shows that without blank column in axial.

$$
B=\left[(1+b)^{2}+\left(1+b^{2}\right)-2 \mu_{d}\left(1-\mu_{d}\right)(1-b)^{2}\right]^{1 / 2} \text {, where, } b \text { is }
$$

the lateral stress coefficient, $b=\frac{\mu_{d}}{1-\mu_{d}}, \mu_{d}$ is the dynamic poisson's ratio of rock; $n$ is pressure increase coefficient of explosive product crashing to hole wall, generally taken $n=10$; $\eta$ is the expansion adiabatic exponent of detonation products, which generally is set to be $3 ; \sigma_{\mathrm{cd}}$ is the uniaxial compressive strength of rock, $\mathrm{MPa} ; \sigma_{\mathrm{td}}$ is uniaxial tensile strength of rock, $\mathrm{MPa} ; r_{b}$ is charge radius. $q$ is charge explosive in unit volume of rock, $\mathrm{kg} / \mathrm{m}^{3}$.

\section{INFLUENTIAL FACTORS OF PRESSURE RELEASING EFFECT OF BLASTING BREAKING ROOF}

\section{A. The influence of charge structure on the pressure releasing effect Charge}

The mechanism of blasting breaking roof shows that the size of fracture zone after blasting is the main factor affecting blasting effect, and it is also the foundation for determining the parameters of blasting technology [14]. Therefore, the influence of charge structure on blasting effect was discussed by analyzing the distribution of crush zone and fracture zone under the different charge structure conditions.

Assuming that rock medium distributes uniformly. The pressure increasing coefficient $n$ of explosive product crashing the hole wall is 10. Expansion adiabatic exponent of the detonation products is 3 . Rock uniaxial compressive strength $\sigma_{\text {cd }}$ is $128.3 \mathrm{MPa}$, while the tensile strength $\sigma_{\mathrm{td}}$ is $6.3 \mathrm{MPa}$. Rock dynamic poisson's ratio is 0.20 , thus $b$ is 0.25 and $B$ is 1.564 . The shock wave attenuation index and stress wave attenuation index were 2.75 and 2.25 respectively. The $\rho_{0}$ is $1.26 \times 10^{3} \mathrm{~kg} / \mathrm{m}^{3}$ (SM II -1 water-gel explosive is $\varphi 32 \times 320 \mathrm{~mm}$, and the weight is $300 \mathrm{~g} /$ volume). Explosive velocity is $1000 \mathrm{~m} / \mathrm{s}$. The relationships between blasting effect with charge uncoupling coefficient $\mathrm{K}$ and charge axial coefficient $l_{e}$ were studied.

Considering the construction factors and the diameter of plastic hose, charge uncoupling coefficient $K$ was selected as $1.2,1.4,1.6,1.8,2.0,2.2,2.4$. Charge axial coefficient was $l_{e}$ $=1$. The change rules of radius of fracture zone and crush zone are shown in figure 2.

When charging axial coefficient $l_{e}$ is $1,0.9,0.8,0.7,0.6$, 0.5 respectively and charge uncoupling coefficient is $K=1.6$, change rules of radius of fracture zone and crush zone are shown in figure 3 .

Figs. 2 and 3 show that the radiuses of fracture zone and crush zone increase with the increase of the charge radius, but the charge radius is limited by bit radius and charge radius, etc. Obviously, the parameters should be selected according to the equipment in coal mine. 


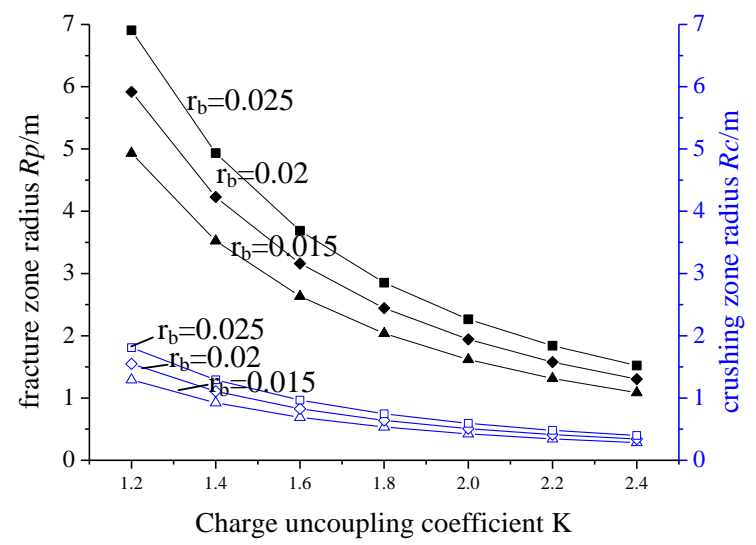

Fig 2 Blasting effect with charge uncoupling coefficient $K$

The uncoupling charge ways, which the hole had a certain distance between charge volume and hole wall to decrease compression shock wave action time and prolong stress wave action time, was selected. Explosion pressure was buffered and explosive power was distributed more uniformly[12]. However, increasing the uncoupling charge coefficient would reduce the energy refraction into the rock[15], leading to less energy used for rock break. The figure 2 shows that radiuses of fracture zone and crush zone decrease with the increase of charge uncoupling coefficient. Therefore, when the uncoupling charge way is used, the charge coupling coefficient still needs to be reduced. Zong gave the impedance matching relations of the uncoupling charge [12]

$$
Z_{m}=\frac{\sqrt{2} K^{-4}}{2} Z_{e}
$$

Where, $Z_{m}$ is the wave impedance of rock, while $Z_{e}$ is the wave impedance of explosive, and $K$ is the charge uncoupling coefficient.

Fig. 3 shows that the radius of fracture zone and crush zone increase gradually with the increase of charge axial coefficient. The continuous charge way is good for expanding the scope of fracture zone and increasing the pressure relief effect, but continuous charge would cause too much energy consumption in crush zone compressed at hole wall, which means it is not good for saving cost. The homogeneous discontinuous charge ways at the blasting cartridge could make better use of blasting energy, and the function of stress wave and detonation gas is better, so the rock breaks more

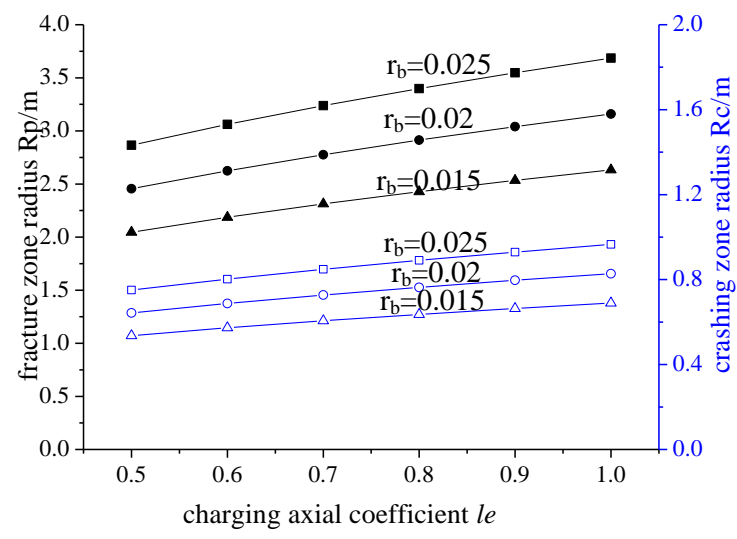

Fig 3 Blasting effect with charge axial coefficient $l_{c}$

uniformly. The impedance matching relations of axial charging was formula (5) [14].

$$
Z_{m}=\frac{\sqrt{2\left(l_{e}-\frac{l_{s}}{l_{b}}\right)}\left(l_{e}-\frac{l_{s}}{l_{b}}\right)^{-4}}{2} Z_{e}
$$

Where, $l_{\mathrm{e}}$ is the charge axial coefficient. $l_{\mathrm{b}}$ is the length of blasting hole. $l_{\mathrm{s}}$ is the sealing length of blasting hole.

\section{B. Simulation Studies of Influence of Blasting Parameters on Pressure Releasing Effect}

The No.3208 working face was chosen as the research object to establish the numerical model by FLAC ${ }^{3 \mathrm{D}}$, the model size was $170 \mathrm{~m} \times 160 \mathrm{~m} \times 77 \mathrm{~m}$. The unit number of calculation model was 126208 , the model boundary conditions were as follows: the horizontal displacement constraint was applied all around, the three directions at the bottom was displacement limited, and the upper boundary was free. According to the $630 \mathrm{~m}$ mining depth, $15.75 \mathrm{MPa}$ weight of overburden stress was applied at the top. The elastic model was used in the model building and Moore Coulomb constitutive model was used in the excavation. Rock mechanical parameters were listed in table 1 .

In the simulation process of blasting breaking roof, the effect of blasting was simulated by reducing the strength of working face and roadway [10], the influence of angles and lengths of blasting holes were researched by weakening different areas. And the influence of different charges on blasting effect was studied through selecting different

\begin{tabular}{|c|c|c|c|c|c|c|c|}
\hline lithology & height (m) & $\begin{array}{c}\text { volume- } \\
\text { weight } \\
\left(\mathrm{kg} / \mathrm{m}^{3}\right)\end{array}$ & $\begin{array}{l}\text { volume modulus } \\
\qquad \text { K (GPa) }\end{array}$ & $\begin{array}{c}\text { shear } \\
\text { modulus } \\
G \quad(G P a)\end{array}$ & $\begin{array}{c}\text { internal } \\
\text { friction } \\
\text { angle ( })\end{array}$ & $\begin{array}{l}\text { Cohesion } \\
\text { (MPa) }\end{array}$ & $\begin{array}{c}\text { tensile } \\
\text { strength } \\
(M P a)\end{array}$ \\
\hline middle-fine sandstone & 20 & 2700 & 6.3 & 5.2 & 40.3 & 4.5 & 3.2 \\
\hline medium mudstone & 5 & 2750 & 8.3 & 7 & 42 & 5.3 & 3.9 \\
\hline mudstone & 3 & 2200 & 4.6 & 3.8 & 33 & 3.6 & 2.0 \\
\hline 2 \#coal seam & 2 & 1350 & 2.1 & 1.78 & 25 & 1.8 & 1.25 \\
\hline medium mudstone & 22 & 2750 & 8.3 & 7 & 42 & 5.3 & 3.9 \\
\hline siltstone & 2 & 2500 & 5.6 & 4.8 & 38.1 & 4.2 & 3.0 \\
\hline 3\# coal seam & 4 & 1350 & 2.1 & 1.78 & 25 & 1.8 & 1.25 \\
\hline medium mudstone & 9 & 2750 & 8.3 & 7 & 42 & 5.3 & 3.9 \\
\hline siltstone & 10 & 2500 & 5.6 & 4.8 & 38.1 & 4.2 & 3.0 \\
\hline
\end{tabular}

TABLE 1 Physical And Mechanical Parameters Of Rock Mass 


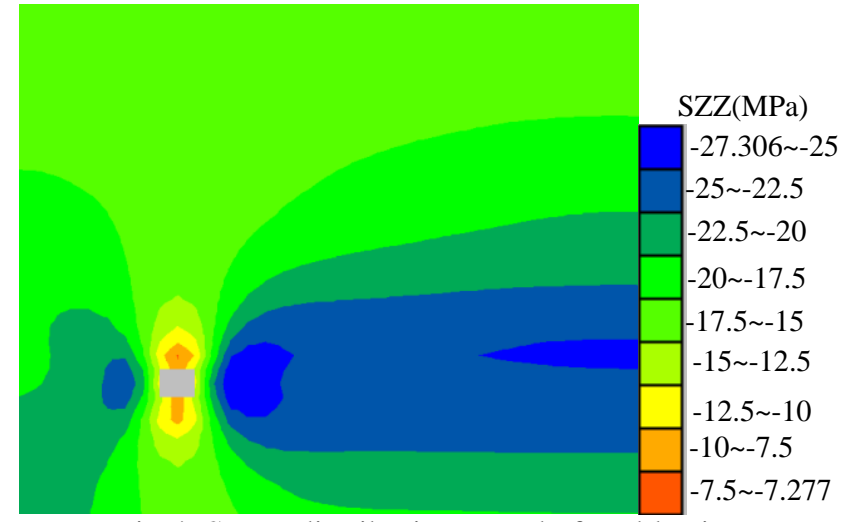

Fig.4. Stress distribution state before blasting

attenuation coefficient $r$ (Figs. 2 and 3 show that the strength in the range of $2.5 \mathrm{~m}$ around the hole was weakened).

\section{1) Influence of Blasting Hole Angle on Pressure Releasing} Effect:

The state of stress distribution of roadway surrounding rock was simulated when the length of blasting hole was $25 \mathrm{~m}$, the weakening degree was $30 \%$, and angles of the blasting hole were $90^{\circ}, 45^{\circ}$ and $0^{\circ}$ respectively. Fig. 4 gives the stress distribution at the $10 \mathrm{~m}$ position in front of the working face before blasting, and Fig.5 gives the stress distribution of different angles of the blasting hole after blasting.

Fig. 4 shows that the excavation of the roadway and the working face leads to stress concentrations at both two side walls of the roadway, and the stress of the coal wall is larger. The maximum concentration stress is $27.31 \mathrm{MPa}$ (the stress concentration factor is 1.73 ) before blasting breaking roof, and the concentration area is large, and the length from the stress concentration area to the roadway wall is about $4 \mathrm{~m}$.

As shown in Fig. 5, after the implementation of blasting breaking roof in roadway, the stress concentration is improved and the distance from the roadway wall increases. When the angle of blasting hole is $90^{\circ}$, the maximum stress is $26.94 \mathrm{MPa}$, just reduced by $1.4 \%$, but the distance from roadway wall to the peak stress area is about $5 \mathrm{~m}$, . When the blast hole angle is $45^{\circ}$, the maximum stress is $26.46 \mathrm{MPa}$. The distance between the coal wall and the peak stress area is about $7 \mathrm{~m}$. When blasting hole angle is $0^{\circ}$, the maximum stress is $26.75 \mathrm{MPa}$ and between the coal wall and the peak stress area is about $6 \mathrm{~m}$. Besides, when the blasting hole angle are $90^{\circ}$ and $0^{\circ}$, the roof and the two coal walls are loose and broken, which can lead to support difficulties.

In conclusion, if the blasting hole length and weakening degree are same, the stress concentration is the smallest and the distance between the coal wall and the peak stress area is the largest when angle of blasting hole is $45^{\circ}$, which means the $45^{\circ}$ angle can get best blasting effect.

2) Influence of Blasting Hole Length on Pressure Releasing Effect

The state of stress distribution of roadway surrounding rock was studied when the angle of blasting hole is $45^{\circ}$, the

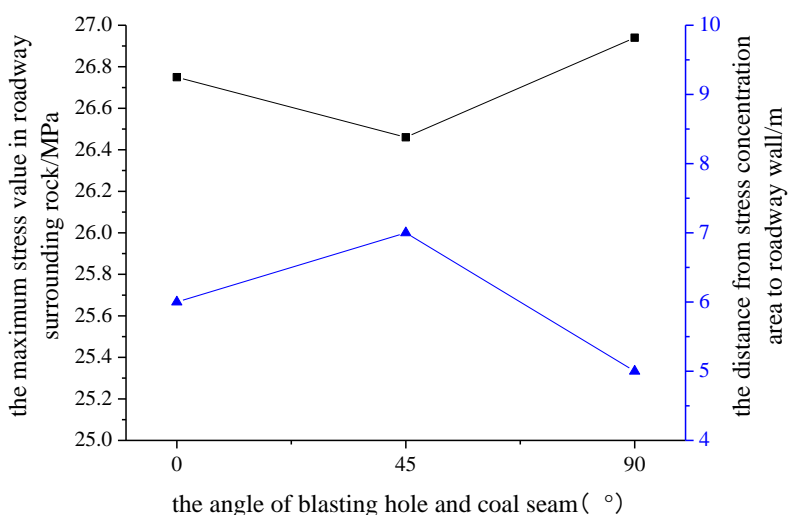

Fig.5. Stress distribution with different blasting hole angle

weakening degree was $30 \%$, while the blasting hole length were $14 \mathrm{~m}, 19 \mathrm{~m}, 25 \mathrm{~m}$, and $32 \mathrm{~m}$, respectively. The influence of blasting hole length on the blasting effect was studied, as shown in Fig. 6.

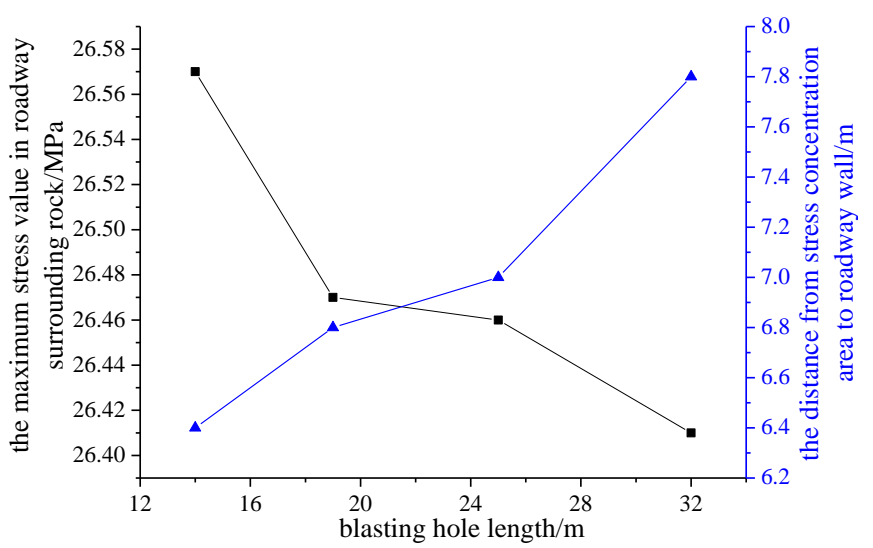

Fig.6. Stress distribution with different blasting hole length

Fig.6 shows that the stress concentration of the surrounding rock decreases and the distance between the coal wall and the peak stress area increases gradually with the increase of blasting hole length. When the blasting hole length increases from $14 \mathrm{~m}$ to $19 \mathrm{~m}$, the stress concentration of surrounding rock decreases obviously, but when the blasting hole length increases from $19 \mathrm{~m}$ to $32 \mathrm{~m}$, the stress concentration and the distance between the coal wall and the peak stress area have little changes. When the blasting hole length increases to $32 \mathrm{~m}$, the peak stress reduces $0.05 \mathrm{MPa}$ and the distance increases $0.8 \mathrm{~m}$, because the blasting hole has gone through the hard rock.

Above all, it shows that the blasting hole length influences blasting effect when the angle of the blasting hole angle and the weakening degree are same. When the blasting hole reaches the stress concentration area, there is no obvious improvement of the blasting effect, but the blasting effect can be improved again when the blasting hole goes across the hard rock. One obvious result can be got is that when the blasting 
hole goes through the hard rock, the pressure releasing effect is the most obvious.

\section{Effect}

3) Influence of Explosive Charge on Pressure Releasing

Different explosive charge has various effects on the weakening degree of rock strength. The influence of different explosive charges on the blasting effect was studied when the weakening degree was $10 \%, 30 \%$, and $50 \%$ respectively, as shown in Fig. 7, while the angle and the length of the blasting hole keeps same, which were $45^{\circ}$ and $32 \mathrm{~m}$, respectively

Fig. 7 shows that the stress concentration of the surrounding rock decreases and the distance between the coal wall and the peak stress area increases gradually with the increase of blasting weakening degree. When the blasting weakening degree is $10 \%$, the peak stress decreases from 27.3MPa to $26.96 \mathrm{MPa}$, and the distance between the coal wall and the peak stress area increases from $4 \mathrm{~m}$ to $5.7 \mathrm{~m}$. When the blasting weakening degree reaches $50 \%$, the peak stress is $25.88 \mathrm{MPa}$, decreasing $1.43 \mathrm{MPa}$. The distance between the coal wall and the peak stress area is $8.5 \mathrm{~m}$, increasing $4.5 \mathrm{~m}$ in depth. Obviously, the blasting effect improves significantly when the explosive charge increases.

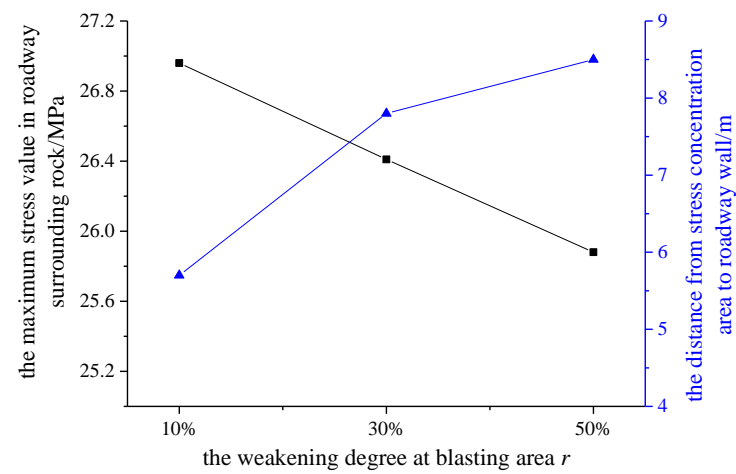

Fig.7. Stress distribution state with different degree of weakening

\section{FIELD APPLICATION}

\section{A. Blasting Parameters and Construction Technology}

Construction equipment and materials: The ZLJ-650 mine roadway drilling rig is used for hole drilling, the diameter of the drilling pipe is $42 \mathrm{~mm}$ and the diameter of the diamond core is $56 \mathrm{~mm}$. The emulsion explosive of coal mine permissibility, whose specification is $\Phi 32 \times 320 \mathrm{~mm}$, and the weight of each explosive is $0.3 \mathrm{~kg}$.Cement cartridge is used to seal hole, whose specification is $\Phi 36 \times 225 \mathrm{~mm}$. Polyethylene plastic pipe was used in the cartridge, whose specification is $\Phi 42 \times 3 \mathrm{~mm}$ (wall thickness). MSB-100 type exploder is used to detonate. Coal mine permitted millisecond delay electric detonators is used and 4 per hole.

Parameter design: The parameters of charge structure and blasting parameters is designed and listed in table 2,and the Blasting hole layout is shown in figure 8 .

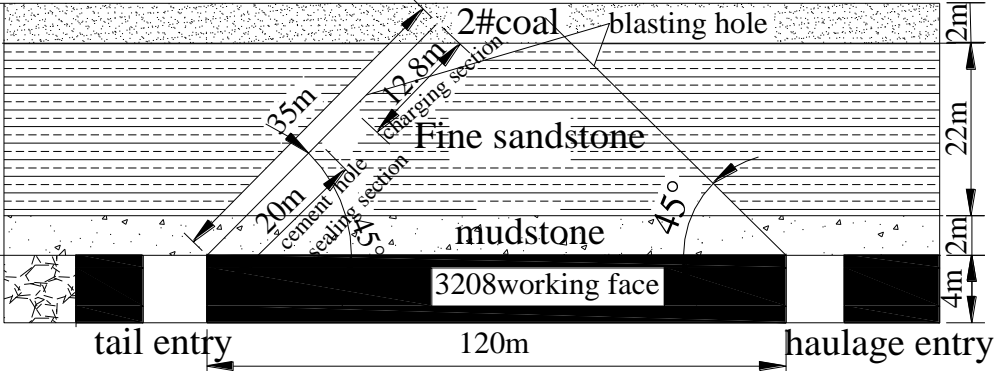

Fig.8. Cross-section drawn of blasting borehole construction in roof

Construction technology: One blasting hole was constructed at the interval of $5 \mathrm{~m}$ in the two roadways from the cut roadway. Positive continuous charge structure without interval was used. The diagram of charge structure is shown in Fig. 9. Detonators were connected in parallel, and cartridges were connected in series, and one to six drilling holes were blasted one time.

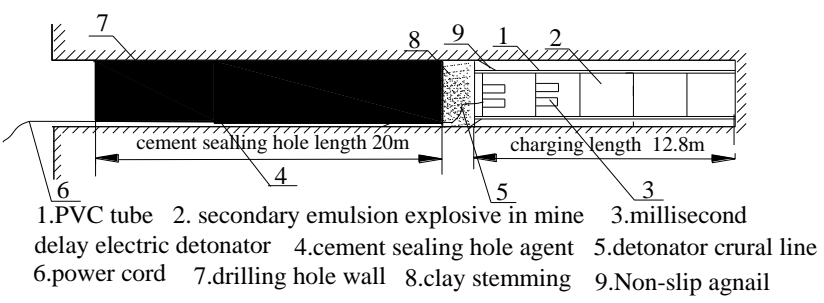

Fig.9. Hole charge structure diagram

\section{B. Application Effect}

Microseismic monitoring method, drilling cuttings method and electromagnetic radiation monitoring method were used during the mining process of No.3208 working face for rock burst monitoring. During the two months of the early production, there were 831 times of microseismic events, one of which had vibration sense and the releasing energy was $9.1 \times 10^{5} \mathrm{~J}$, but no abnormal pressure showed. The microseismic events were listed in table 3 :

TABLE2. Parameters Of Charge Structure And Blasting Parameters

\begin{tabular}{cccc}
\hline hole diameter & $56 \mathrm{~mm}$ & length of explosive charge & $12.8 \mathrm{~m}$ \\
\hline charge radius & $16 \mathrm{~mm}$ & length of cement hole sealing & $20 \mathrm{~m}$ \\
charge uncoupling coefficient & 1.75 & charge axial coefficient & 0.85 \\
angle of blasting hole & $45^{\circ}$ & length of blasting hole & $35 \mathrm{~m}$ \\
spacing of blasting hole & $5 \mathrm{~m}$ & explosive charge & $12 \mathrm{~kg}$ \\
\hline
\end{tabular}


TABLE 3. Microseismic Events Of 3208 Working Face

\begin{tabular}{|c|c|c|}
\hline Energy level 0) & Times & The proportion \\
\hline $\mathrm{E}<10^{4} \mathrm{~J}$ & 804 & $96.8 \%$ \\
\hline $\mathrm{E} \geq 10^{4} \mathrm{~J}$ & 27 & $3.2 \%$ \\
\hline summation & 831 & \\
\hline
\end{tabular}

The microseismic events whose energy was less than $10^{4} \mathrm{~J}$ accounting for $96.8 \%$ of the total in the No.3208 working face, which indicates that most of the microseismic events released small energy. Overall, the probability of rock burst in No.3208 working face was small, and the blasting effect was good.

\section{CONCLUSION}

The theoretical analysis, numerical simulation and field application were used to study the influencing factors of the pressure releasing effect of blasting breaking roof, and the main conclusions are as follows:

1) Crush zone, fracture zone and vibration zone are formed around the blasting hole after the blasting to break hard roof. The mechanical properties of the coal and rock are weakened due to the damage of coal in the crush zone and the fracture zone. Thus the coal loses the ability to accumulate high stress and store high elastic deformation energy. Obviously the radiuses of crush zone and fracture zone can be used as evaluation indexes of blasting effect.

2) Charge uncoupling coefficient $K$, charge axial coefficient $l_{e}$, and charge radius $\mathrm{r}_{\mathrm{b}}$ has obvious influences on the blasting effect. The pressure releasing effect increases with the increase of charge radius. On the premise of using uncoupling charge way, reducing the charge uncoupling coefficient is good for improving the pressure relief effect. Continuous charge ways are beneficial for expanding the scope of fracture zone and increasing the pressure relief effect. The best impedance matching relationship of uncoupling charge and axial charge are also given.

3) Numerical simulation results show that the stress concentration of surrounding rock is the minimum and the distance between the coal wall and the peak stress area is the maximum when the angle of the blasting hole is $45^{\circ}$. When the blasting hole goes through the hard rock, the effect of breaking roof to relief pressure is better. The larger the blasting weakening degree is, and the more the explosive charge is.

(4) Based on the research results, and the equipment and materials of one coal mine, the parameters of charge structure and blasting parameters were designed: the charge uncoupling coefficient is 1.75 , the charging axial coefficient is 0.85 , the charge radius is $0.016 \mathrm{~m}$, the $5 \mathrm{~m}$ blasting spacing is $5 \mathrm{~m}$, the angle of blasting hole is $45^{\circ}$, the length of the blasting hole is $35 \mathrm{~m}$ and explosive charge is $12 \mathrm{~kg}$. The field monitoring results show that the application effect is good.

\section{ACKNOWLEDGMENT}

The research described in this paper was financially supported by National Natural Science Foundation of China (No.51474136, No.51474137, No.51374140 and No.51274133), Shandong Province Science and Technology Development Plan Item (No.2014GSF120002), Tai'shan Scholar Engineering Construction Fund of Shandong Province of China, Qingdao Postdoctoral Applied Research Project (No. 2015198), China Postdoctoral Science Foundation Funded Project (No.2016M592220).

\section{REFERENCES}

[1] Yunliang Tan, Shiliang Wu, Zengde Yin et al, Mining pressure and strata control[M]. Coal industry publishing house Beijing, China, 2008.

[2] Yunliang Tan, Xueshui Wang. A Preliminary study of acoustic emission of rock during the roof weighting process in a coal mine $[\mathrm{J}]$. Chinese Journal of Rock Mechanics and Engineering, 1992,11(3):275-283

[3] Yunliang Tan, Ze Zhang, Tongbin Zhao. AE Pattern of Rock Burst Disaster Induced by Strata Activation in Coal Mine[J]. Disaster Advances, 2011, Vol. 4 (4): 29-33.

[4] Yunliang Tan, Ze Zhang. Rock Burst Disaster Induced by Mining Abutment Pressure[J]. Disaster Advances, 2012 ,5(4):378-382.

[5] Tongbin Zhao, Yanchun Yin, Yunliang Tan. Safe mining and new prediction model in coal seam with rock burst induced by roof[J].Disaster Advances, 2012,5(4):691-695.

[6]Yongliang Yang, Daopin Cui, Yage Li. Relieving-shot of controlling the deformation of roadway[J]. Ground Pressure and Strata Control, 2005, (1): 33-35.

[7] $\mathrm{Xu} \mathrm{Lu}$, Feng Zhang, Shaohua Zhou. Application of digital simulation to vibration blasting and pressure releasing technology [J]. Coal Science and Technology, 2005, 33(8): 21-23.

[8] Mingtao Gao, Yuying Wang. Study and application on the technology of using the blasting to break roof to control rock burst[J]. Journal of China coal society, 2011,36 (s2):326-331.

[9] Zuqiang Xiong, Huaijian He. Numerical simulation of rock burst stress and its control by stress-relief[J]. Journal of Mining and Safety Engineering, 2006, 23(4):489-493.

[10] Linsheng Zhou, Kegong Fan, Jun Liu, et al.Numerical simulation of the effect on pressure relief by blasting in working face with rock burst[J]. Journal of Shandong University of Science and Technology, 2005,24(4): 77-80.

[11] Qingxin Qi, Yi Lei, Hongyan Li, et al. Deep- hole blasting in top rock to rock burst prevention theory and practice[J]. Chinese Journal of Rock Mechanics and Engineering, 2007, 26 (S1):3522-3527.

[12] Shankun Zhao, Yongren Wang, Baoyang Wu, et al. Numerical Simulation and Application of Rock Burst Prevention by Advanced Deep- hole Blasting[J]. Chinese Journal of Underground Space and Engineering 2015, 11(1):89-97.

[13] Mingyao Wei, Enyuan Wang, Xiaofei Liu,et al. Numerical simulation of rockburst prevention effect by blasting pressure relief in deep coal seam[J]. Rock and Soil Mechanics,2011, 32(8):2539-2543.

[14] Qi Zong. Influence of charging construction on energy transmission of explosion[J].Journal of Fuxin Mining Institute (natural science), 1997,16(1):51-54

[15] Qi Zong, Dejun Meng. Influence of different kinds of hole charging structure on explosion energy transmission[J]. Chinese Journal of Rock Mechanics and Engineering, 2003,22 (4) :641645. 